\title{
The social basis of referential communication: Speakers construct reference based on listeners' expected visual search.
}

\author{
Julian Jara-Ettinger ${ }^{1}$, Paula Rubio-Fernandez $z^{2,3}$ \\ ${ }^{1}$ Department of Psychology, Yale University \\ ${ }^{2}$ Department of Philosophy, Classics, History of Art and Ideas, University of Oslo \\ ${ }^{3}$ Department of Brain and Cognitive Sciences, MIT
}

\begin{abstract}
A foundational assumption of human communication is that speakers should say as much as necessary, but no more. In referential communication, the pressure to be efficient is typically formalized as an egocentric bias where speakers aim to minimize production costs. While intuitive, this view has failed to explain why people routinely produce redundant adjectives, particularly color words, or why this phenomenon varies cross-linguistically. Here we propose an alternative view of referential efficiency, whereby speakers create referential expressions designed to facilitate the listener's visual search for the referent as they process words in real time. We present a computational model of our account, the Incremental Communicative Efficiency (ICE) model, which generates referential expressions by considering listeners' expected visual search during online language processing. Our model captures a number of known effects in the literature, including cross-linguistic differences in speakers' propensity to over-specify. Moreover, our model predicts graded acceptability judgments with quantitative accuracy, systematically outperforming an alternative, brevity-based model. Our findings suggest that reference production is best understood as driven by a cooperative goal to help the listener identify the intended referent, rather than by an egocentric effort to minimize utterance length.
\end{abstract}

Key words: Computational modeling, Social cognition, Pragmatics, Reference

\section{Introduction}

According to Zipf's Principle of Least Effort, languages are shaped by the competing pressures of minimizing production costs for the speaker while maximizing ease of comprehension for the listener (Zipf \& Behavior, 1949) - an idea that goes back to von der Gabelentz's (1901) balance between 'ease' and 'clarity' (cited in Fedzechkina \& Jaeger, 2020). The Gricean Maxim of Quantity identifies analogous communicative pressures in language use: speakers should provide their listeners with as much information as required, but not more (Grice et al., 1975). That is, successful communication requires speakers to produce unambiguous messages while remaining succinct. This maxim has been highly influential in pragmatic theories of communication because it reflects an assumption that listeners make about their conversational partners, enabling them to go beyond the literal meaning of a message. Imagine, for example, that your partner asks you to pass them 'the plastic mug.' Holding your partner to the expectation that they produced the shortest sufficient description would allow you to infer that there are several mugs of different materials to choose from, and that 
your partner has a specific one in mind. Similarly, if, in the same situation, your partner had only requested 'the mug', you could conclude that they were unaware there were multiple mugs available.

Despite the maxim's intuitive appeal, quantifying the amount of information necessary to convey a message, or the amount of redundancy contained in different messages, is far from straightforward. In the above situation, suppose that 'the plastic mug', 'the red mug', and 'the red plastic mug' all uniquely identify your partner's mug. A brevity analysis would directly rule out the longer description; but what about choosing between color modification or material modification? This basic instance of referential communication (the typical domain where the Maxim of Quantity has been studied; Sedivy 2003, 2005; Pogue et al. 2016; Ryskin et al. 2019; Engelhardt et al. 2006, 2011; Davies \& Katsos 2013), shows how a brevity interpretation of the Gricean maxim fails to distinguish between descriptions matched in word length (e.g., 'the plastic mug' vs 'the red mug'), which may nonetheless differ according to other metrics of communicative efficiency.

Here we propose an alternative interpretation of this maxim, which rests on the idea that speakers and listeners share the same goal in interactive referential communication: namely, to ensure that the listener identifies the intended referent with limited expenditure of time and effort. Given this joint goal, an efficient referential expression should not only provide sufficient information to avoid ambiguity, but also facilitate the listener's visual search for the referent. Under this formulation, cost estimation is not entirely egocentric (i.e. determined by the speaker's production costs), but is in fact partly allocentric (i.e. aimed to minimize costs for the listener). That means that amongst two equally informative descriptions, the more efficient one would help the listener identify the referent faster than the other one. According to this metric of efficiency (and leaving aside other considerations, such as word frequency, for example), in a situation where 'the red mug' and 'the plastic mug' are equally informative, an efficient speaker should prefer the color description over the material description because color is normally easier to discriminate than material.

For speakers, deciding what information will be most helpful to listeners requires more than simple perspective taking (in terms of what they can or cannot see) and involves considering how their visual perception (e.g., what is visually salient and what is not) will guide their visual search during real-time language processing (Eberhard et al., 1995; Spivey et al., 2001; Rubio-Fernandez \& Jara-Ettinger, 2020). Upon hearing, 'the red mug', for instance, English speakers begin their visual search guided by color and then narrow it down by kind. This implies that listeners can begin with an easy perceptual task (identifying red objects in a visual scene) and then engage in a harder one (identifying mugs; Rubio-Fernandez 2020; Rehrig et al. 2020). By contrast, when Spanish speakers hear the equivalent expression 'la taza roja' (the mug red), they begin their visual search guided by kind, and then narrow it down by color (inverting the order in the relative ease of visual search; Rubio-Fernandez et al. 2020). Thus, under our account, speakers must be sensitive to listener's expected visual search as a function of the visual context, the target referent, and the language's word order.

A growing body of recent empirical work has discovered patterns of linguistic preference that are difficult to explain through a brevity-interpretation of the Gricean Maxim of Quantity (and we review these effects in detail below). These studies support the idea that reference production is shaped by a goal to facilitate processing for the listener, rather than minimize production costs for the speaker (Rubio-Fernandez, 2019; Rubio-Fernandez et al., 2020; Rubio-Fernández, 2016; Long et al., 2020; Rehrig et al., 2020; Sonnenschein \& Whitehurst, 1982; Mangold \& Pobel, 1988; Paraboni et al., 2007; Paraboni \& Van Deemter, 2014; Arts et al., 2011; Tourtouri et al., 2019). However, no work to date has formalized this idea in precise computational terms. We therefore lack a computational theory that clearly specifies the computations and cognitive capacities that might underlie an allocentric metric of efficient communication. Here we present a computational model that formalizes the idea that speakers generate expressions that are not only sufficiently informative, but also minimize the 
listener's time and effort to identify the referent. We validate the principles behind our model empirically, comparing speakers' referential preferences with listeners' visual search, and we test its predictions in a quantitative manner against existing reference production data, and in a novel acceptability task designed to test our model in a rigorous and comprehensive manner.

The paper is structured as follows. We begin by reviewing classical frameworks to model reference (Section 2). We then review recent empirical evidence that highlights challenges to the standard interpretation of the Maxim of Quantity in terms of brevity, and we introduce the incremental efficiency theory, which posits that speakers aim to generate referential expressions that help listeners efficiently identify the referent, under the assumption that they process language incrementally (Section 3). We then turn to our main contribution. We present the Incremental Communicative Efficiency (ICE) model, which produces referential expressions by considering listeners' real-time visual search through a purely allocentric cost function (Section 4). In this section we also review recent advances in modeling referential over-specification, and we discuss how these models relate to our approach. Finally, we show how our model explains production and acceptability judgments better than a brevity model with egocentric costs, and that it unifies a range of empirical phenomena around over-specification and cross-linguistic variation (Sections 5-8). We end with a discussion of the implications of our results on our understanding of reference production, and on the interaction between communication and Theory of Mind (Section 9).

\section{Computational Models of reference}

In recent years, the Gricean Maxim of Quantity has been formally and informally operationalized as the construction of an utterance that simultaneously maximizes the probability that a listener identifies the intended message in a semantic space (i.e. making the message as informative as required) while minimizing its production cost (i.e. while saying no more). Formally, given an intended meaning $m$, the Maxim of Quantity can be implemented as the process of selecting a utterance $u$ that maximizes the utility function

$$
U(u, m)=f\left(p_{L}(m \mid u)\right)-C(u)
$$

where $U(u, m)$ is the utility of producing utterance $u$ to communicate meaning $m . p_{L}(m \mid u)$ is the probability that a listener would identify meaning $m$ upon hearing utterance $u$, and $f$ is a measure of communicative success that depends on on this probability. For instance, $f$ might be a binary indicator of whether the utterance is unambiguous, or it could encode the information-theoretic measure of surprisal (Kao et al., 2014; Degen et al., 2019; Goodman \& Frank, 2016). The final term, $C(u)$, expresses the cost associated with producing utterance $u$.

Under this framework, the first term- $f\left(p_{L}(m \mid u)\right.$ - captures the idea that speakers ought to be sufficiently informative. Returning to the example from the introduction, an ambiguous expression like 'the mug' would have a low utility because it the speaker would have a lower probability of identifying the right mug, relative to when they hear utterances like 'the red mug', 'the plastic mug', 'the red plastic mug', or even relative clauses, such as 'the mug that is red and made of plastic'. While this first terms identifies under-informative utterances, it fails to distinguish between utterances that are sufficiently informative, like 'the red mug' and 'the mug made of plastic'. The second term implements the pressure to be succinct. Thus, the cost of production would favor 'the red mug' and 'the plastic mug' because they ensure communicative success in shorter utterances relative to 'the red plastic mug' or 'the mug that is red and made of plastic'.

This general approach has enjoyed wide success in capturing referential communication in both speakers and listeners (Frank \& Goodman, 2012; Goodman \& Frank, 2016; Degen et al., 2019; JaraEttinger et al., 2019; Kao et al., 2014) and it's utility-based formulation shares a similar structure 
to non-linguistic social inferences that, from infancy, structure how people reason about each other's goals and mental states (Jara-Ettinger et al., 2016; Bridgers et al., 2020; Lucas et al., 2014; Liu et al., 2017).

While the role of information gain and its tradeoff with costs have received substantial theoretical and empirical attention, this framework also implicitly posits a conceptual distinction between the two processes: the pressure to be sufficiently informative is allocentric-determined by considering how a listener might interpret an utterance - while the pressure to be brief is egocentric - estimated as the speaker's effort in producing an utterance, with no regard for a listener's perspective. Indeed, while the vast majority of models of reference production implement an egocentric utterance cost, the distinction between egocentric and allocentric costs is rarely discussed, explicitly adopting an agnostic attitude to what costs might look like in complex situations (Kao et al., 2014; Degen et al., 2019; Krahmer et al., 2003).

\section{The incremental efficiency theory}

Under the framework presented above, a standard interpretation of the Gricean Maxim of Quantity as one of brevity would dictate that the cost function $C(u)$ ought to be egocentric and penalize utterances based on length. This formalization, however, faces two empirical challenges in referential communication. First, people often produce redundant adjectives, especially color (Arts et al., 2011; Rubio-Fernández, 2016; Rubio-Fernandez, 2019; Sedivy, 2003, 2005; Koolen et al., 2013). For example, your partner may ask you for 'the red mug', even if there is only one mug in the scene. Second, English speakers tend to produce more redundant color adjectives than Spanish speakers when referring to the same objects in the same displays (Rubio-Fernández, 2016; Rubio-Fernandez, 2019; Rubio-Fernandez et al., 2020). The brevity interpretation of the Gricean maxim fails to explain why people sometimes choose to use redundant adjectives, nor why speakers of languages with prenominal modification (such as English; e.g., 'the red mug') should use color adjectives more frequently than speakers of languages with postnominal modification (such as Spanish; e.g., 'la taza roja') in situations where color is equally overinformative.

The standard view also faces two theoretical challenges. First, the brevity maxim does not distinguish between referential expressions of equal length that may be equally informative, but not necessarily equally efficient (particularly with respect to the listener, e.g., 'the red mug' vs 'the plastic mug'). Second, it fails to take into account incrementality - a universal pressure that shapes pragmatics across languages (Rubio-Fernandez \& Jara-Ettinger, 2020) — analyzing utterances globally rather than incrementally. Such an analysis fails to distinguish 'the red mug' and 'la taza roja', contrary to empirical evidence that the two expressions are not treated as equivalent by speakers and are processed differently by listeners (despite having the same meaning in English and Spanish; Rubio-Fernandez et al. 2020). This latter challenge is highly relevant for computational models of reference production, which, to the best of our knowledge, have only been built for languages with prenominal modification, such as English or Dutch (e.g., van Gompel et al., 2019; Degen et al., 2019), even though most world languages have postnominal modification (Dryer et al., 2005).

The alternative view of referential communication that we propose here - the incremental efficiency theory - posits that speakers aim to produce referential expressions that are incrementally efficient for listeners. That is, an expression's efficiency ought to be computed by evaluating how it guides listeners towards the intended message as they process words incrementally. This theory predicts that, amongst equally informative descriptions, speakers should prefer the one that ensures communicative success most efficiently. In the case of referential communication, this should lead to a preference for encoding visually salient properties, to the extent that they help the listener find 
the object more efficiently (e.g., favoring 'the red mug' over 'the plastic mug'), given the complexity of the visual scene and the language's word order. Thus, this view of referential communication naturally integrates an expression's informativity (e.g., which properties may uniquely identify the mug you want to request), perceptual factors (e.g., whether the mug you want is the only red object on the table so red is perceptually salient) as well as incrementality (e.g., whether you will formulate your request in English or in Spanish; Rubio-Fernandez et al. 2020).

To date, virtually all analyses of referential informativity, either theoretical (Sedivy, 2003, 2005; Engelhardt et al., 2011) or computational (Krahmer \& van Deemter, 2012; van Deemter et al., 2012; Gatt et al., 2014), have calculated informativity relative to the full description (e.g., 'the red mug'; although see Cohn-Gordon et al. 2018 for a recent exception) and the number of objects of the same kind as the target (which could cause ambiguity if the speaker did not distinguish them; e.g., other mugs in the scene). According to the standard view, the referential expression 'the mug' would be optimally informative if there was a single mug in the visual context, whereas it would be underinformative if there was more than one mug. Likewise, 'the red mug' would be optimally informative if there were several mugs and only one was red, but it would be overinformative if there was only one mug.

According to the incremental efficiency theory, and contrary to the standard analysis, the efficiency of a referential expression needs to be established incrementally, rather than globally, and relative to the entire visual context, rather than only with respect to those objects of the same kind as the target. Thus, the relative efficiency of the prenominal adjective 'red' should be evaluated based on the incomplete phrase 'the red ...' rather than on the full description 'the red mug'. Under this analysis, the color adjective may be an efficient referential choice if it secures unique reference for the listener, regardless of the number of mugs in the scene (e.g., if the red mug is the only red object on the table). Likewise, even if there were several mugs of different colors and the full description 'the red mug' established unique reference, the incomplete phrase 'the red ...' may nonetheless result in a temporary ambiguity if other objects in the visual context are red. In such a situation, 'the plastic mug' may turn out to be a more efficient referential expression if the material adjective secured unique reference with less processing effort for the listener.

The incremental efficiency theory is therefore novel on two accounts. First, it conceptualizes the relative efficiency of a referential expression incrementally and across the entire visual context (rather than globally and only with respect to those objects of the same kind as the target). Second, it treats utterance costs allocentrically, capturing the estimated difficulty of the listener's visual search for the referent (rather than egocentrically, as the speaker's production costs measured in number of words). While recent work has argued about the importance of incrementality and efficiency in communication (Rubio-Fernandez \& Jara-Ettinger, 2020), particularly with respect to over-specification (Rubio-Fernandez et al., 2020), no work to date has formalized the incremental efficiency theory. This was the aim of the present study. We present a precise and quantitative formulation of the incremental efficiency theory, implemented through an allocentric notion of efficiency, and we test the theory's explanatory power in a quantitative manner.

\section{Computational Framework}

Model code is available on our project's OSF repository, and additional information is available in our supplemental materials. To illustrate the logic of the Incremental Communicative Efficiency (ICE) model, Figure 1 shows examples of the types of situations that we consider. Each event consists of a grid of objects, one of which is the target referent (surrounded by a gray border). Throughout, we consider four different ways to describe the target: by providing the noun, by providing the noun accompanied by either its material or its color, and by providing the noun accompanied by both its 
material and color. We do not consider other properties that speakers could rely on (such as texture, shape or size), although our framework can be trivially extended to include additional features. We return to this point in the discussion.

We take as a starting point the utility-based frameworks that capture human commonsense psychology (Jara-Ettinger et al., 2016, 2020; Liu et al., 2017) and referential communication (Degen et al., 2019; Goodman \& Frank, 2016; Frank \& Goodman, 2012). Our model extends these frameworks using two driving principles: First, we compute the utility of an expression as it unfolds over time and listeners process it incrementally. Second, we do not conceptualize speaking as a fundamentally costly activity. Instead, we assume that speakers are motivated to minimize the time it takes listeners to find the target, thus attempting to provide expressions that are structured to help the listener identify the referent as quickly as possible.

Formally, given a target object $t$ in a visual scene, our model estimates the utility of different referential expressions, $U_{t}(r)$, and then assigns a probability to each expression by softmaxing this utility function, such that the probability associated with referential expression $r$ is given by

$$
p(r) \propto \exp \left(U_{t}(r) / \tau\right)
$$

where $\tau \in(0, \infty)$ is the softmax parameter, which determines the speaker's capacity to identify the utterance with the highest utility. When $\tau$ is low, softmaxing captures a speaker who consistently identifies and selects the expression with the highest utility, even when alternative expressions have comparably high utilities. As $\tau$ increases, the softmaxing begins to probabilistically select expressions that have lower utilities, but continuing to favor expressions with higher utilities.

We define the utility of referential expression $r$ to communicate target $t$ as

$$
U_{t}(r)=R(t) p_{L}(t \mid r)-C(T ; t, r) .
$$

$p_{L}(t \mid r)$ is the probability that the listener will correctly identify the target $t$ upon hearing referential expression $r$, and $R(t)$ is the speaker's subjective reward for successfully communicating target $t$. Thus, their product captures the referential expression's expected reward. We define $p_{L}(t \mid r)$ as $1 / n$, where $n$ is the number of objects in the visual scene that are consistent with expression $r$. For example, in Figure 1a, the expressions 'the hat', 'the black hat', 'the woolen hat', and 'the black woolen hat' all assign probability 1 to the hat. By contrast, in Figure 1c, the expressions 'the box', and 'the black box' only assign a probability of 0.5 to the target, because the display contains two black boxes. However, the expressions 'the cardboard box' and 'the black cardboard box' assign a probability of 1 to the target, because the second box is made of wood (not cardboard).

The second term, $C(T ; t, r)$, is a measure of the expected time $T$ that it will take the listener to identify a valid target $t$ as they process the referential expression $r$. Thus, this term captures the key idea of an allocentric cost where the speaker is motivated to convey the meaning to the listener in an efficient manner. To compute this value, we provided our speaker model with a simple model of listeners' visual search.

\subsection{Listener visual search model}

Our visual search model captures the expectation that listeners integrate the meaning of each word as they hear it, and use it to guide their visual search for the referent. To our knowledge, no empirical work has characterized this intuitive theory of human visual search. Our visual search model therefore aims only to approximate this intuitive theory, allowing us to formalize and test the incremental efficiency theory. However, this initial model is likely to miss nuances in visual search that may make its predictions more nuanced. We return to this point in the discussion. 

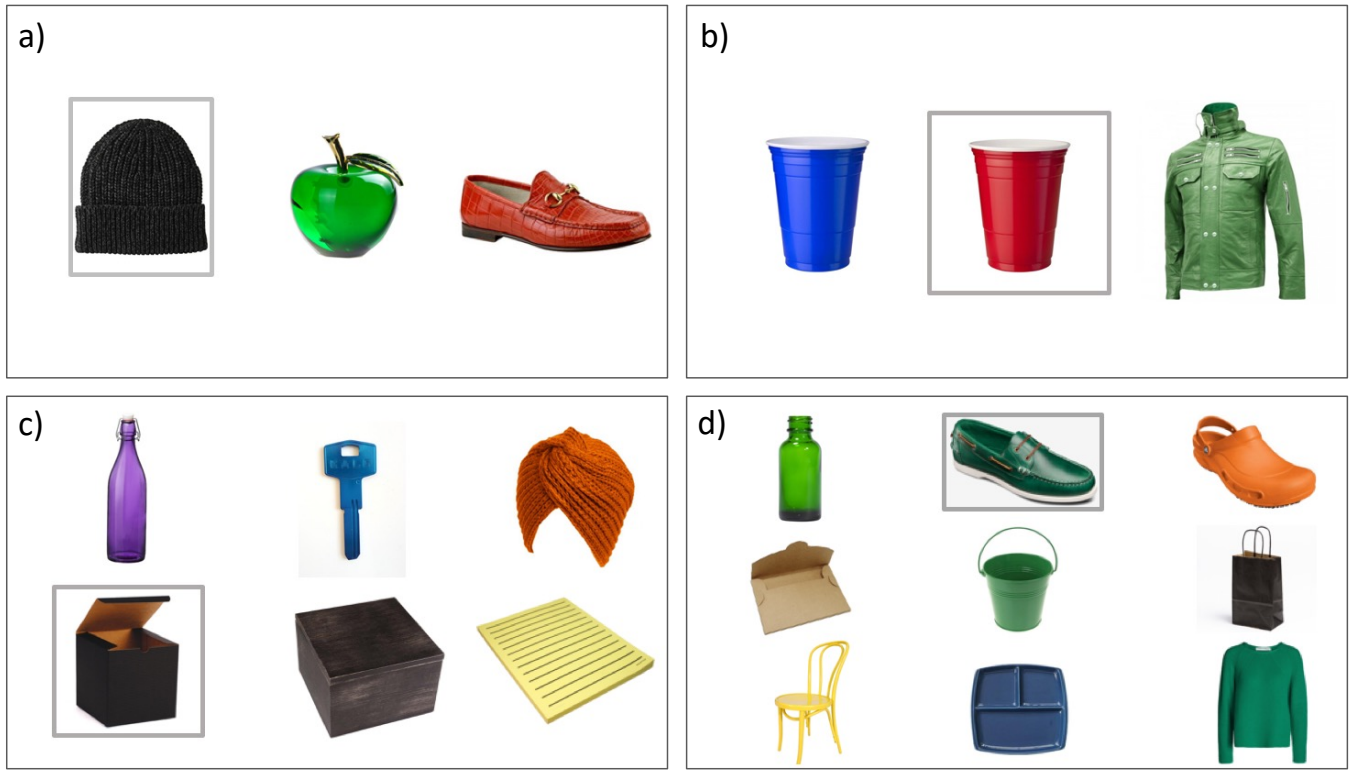

Figure 1: Sample displays from the model test. Displays varied in the number of objects $(3 / 6 / 9)$ and the presence or absence of a contrast object. For example, in Display A, the target is the only hat (which makes modification redundant), whereas in Display B, there are two plastic cups (which makes color modification necessary to identify the target). The target and the contrast object differed either by color (Display B), material (Display C), or both (Display D). Displays also varied in the number of color or material competitors of the target. For example, in Display $\mathrm{D}$, the target is one of four green objects. 
Given a visual scene, our model begins searching for the object as soon as the speaker utters the first content word in a referential expression (for simplicity, we do not process the word 'the', as it does not discriminate the target in the contexts that we consider here). During this search, the model always attempts to locate objects in the scene that match the description heard so far. For instance, upon hearing 'the red ...' or 'the plastic ...' our model searches for red objects or plasticmade objects, respectively. Next, when hearing the noun, e.g., 'basket', the speaker integrates this information and updates the search for red baskets or plastic baskets, respectively.

To model this search process, we implemented a distinction between object properties that listeners can detect from the visual periphery and object properties that they can only evaluate by fixating on an object. Specifically, we treated color as a property that listeners can detect from the periphery, without having to fixate on the object, and we treated material and category as properties that are only revealed by directly fixating on an object (see Section 5 for an empirical validation of this assumption).

Equipped with this distinction, our visual search model could either fixate on a random object and evaluate its properties, or direct fixations to objects with any property that could be evaluated peripherally. That is, in the context of our task, the visual search model could either choose a random object to fixate on (and thus recognize its material and category), or direct the fixation to an object of any desired color (as color can be detected from the periphery, recognizing its material and category once the model has fixated on it). Thus, when a target's color is available, as when processing expressions like 'the red ...', 'the red plastic ...', or 'the red plastic mug ...', our model searches for the target by always directing fixations to red objects, and evaluating the object based on the additional information included in the description (e.g., testing whether the fixated red object is made of plastic when the partial expression is 'the red plastic ...'). By contrast, when only nonperipheral properties are available, such as when processing expressions like 'the plastic ....', 'the plastic chair', or 'the chair', our model, unable to detect material or category from the periphery, would search for the target by fixating on random objects and evaluating their properties (but not fixating again on objects it had already discarded).

During this online search, whenever the model fixates on an object that does not match the expression, it would continue searching for the target (e.g., if the model fixated on a red metal object after hearing 'the red plastic ...', it would redirect its fixation to another red object that it had not evaluated yet). However, if the model fixated on an object that matched the expression heard so far, it would temporarily hold its fixation and wait to obtain more information (e.g., in processing the description 'the red plastic ...', the model would hold its fixation if it found a red plastic object, waiting to find out the category and see if it matched), or select the object if the expression was complete (e.g., if it were fixating on a red plastic chair after hearing 'the red plastic chair').

The speed with which the search model identifies a referent depends on the linguistic information available (and the order in which the speaker makes it available, as determined by their language's word order), the complexity of the visual scene, and the visual salience of the properties encoded in each word. In addition, due to the probabilistic nature of our model, the same expression in the same visual context can nonetheless produce variable search times, depending on whether the visual search model happens to fixate on the right objects early in the search or not. Thus, in our complete model, we approximated the expected number of fixations required to identify the target through 10000 visual search simulations. We then used this estimate as a proxy for the expected time a listener engages in visual search for any referential expression in any visual context. We thus see this component as a way to capture speakers' broad intuitions about the relative ease or difficulty of visual search, without claiming that the speaker actively quantifies listeners' search cost in terms of fixations. 


\subsection{Alternative brevity account}

To better understand how our model behaves relative to a standard brevity interpretation of costs, we also implemented an alternative model where the utility of a referential expression is given by

$$
U_{t}(r)=p_{L}(t \mid r) R(t)-C(r)
$$

This model has the same structure as our ICE model (Eq. 3, differing only in that we replace the allocentric cost function with an egocentric one, $C(r)$, which assigns a cost based on utterance length, estimated in number of words. Combined, the first term leads the speaker to produce expressions that uniquely identify the target, while the second term incentivizes the speaker to favor shorter expressions over longer ones.

\subsection{Model parameters}

Our ICE model has three parameters: the reward associated with communicating successfully $(R(t))$, the softmax parameter $(\tau)$, and the rate of fixations per word (FPW). The FPW parameter that specifies the speed of fixations relative to the speed of speech. Thus, a FPW of 1 would mean that the rate of fixations matches the rate of words, while a higher FPW would mean the listener can perform multiple fixations for each word that the speaker produces.

Similarly, the Brevity alternative model also has three parameters. The first two are identical to the ones from our main model: a reward associated with communicating successfully $(R(t))$, and a softmax parameter $(\tau)$. However, rather than having a fixations per-word parameter in the cost function, the alternative model has a parameter that determines the cost of producing each word (WC).

The first parameter - the reward for communication - was introduced to ensure that both models place a high motivation to communicate unambiguously. This parameter effectively serves as a constant scaling factor that ensures that the models never produce the unnatural situation where a speaker might be intentionally ambiguous and forego communicative success for the sake of incurring lower costs. For both models, we treated this parameter as a fixed constant independent of the target, set to $R(t)=30$ for the ICE model and $R(t)=1$ for the Brevity model (prior to data collection). ${ }^{1}$

The remaining two parameters of the ICE model were also set prior to data collection. For simplicity, we assumed an equal speed of fixations and speech $(\mathrm{FPW}=1)$. We next set the softmax parameter to $\tau=2$, chosen qualitatively to ensure that the model produced probability distributions with variable strengths of beliefs (such that they could be correlated with participant judgments; a $\tau$ level too low would produce distributions that place all weight on the highest utility with no variability, while a $\tau$ too high would produce uniform distributions with no information about the underlying utilities).

To make the models comparable, we next aligned the alternative Brevity model with the ICE model, such that their relative degrees of confidence and uncertainty were matched (e.g., if the ICE model was fully confident about which utterance to select on $n$ of the trials in our experiments, then the Brevity model should also be confident on $n$ of the trials, although the trials where each model is confident would naturally be different ones). This enables us to compare the models directly while removing the possibility that one model might outperform the other due to differences in confidence. To achieve this alignment, we generated our ICE model's predictions for the stimuli used in our main experiment and we then found the Brevity model parameters that matched the range of ICE model predictions as closely as possible (using an euclidean measure between the two probability

\footnotetext{
${ }^{1}$ Note that the ICE model can have higher higher costs (which index fixations) relative to the Brevity model (which counts words weighted by a lower parameter value, as we discuss below), motivating the setting of a higher reward.
} 
distributions; see Supplemental Materials for details). This resulted in a word cost of $W C=0.09$ and $\tau=0.12$ (note that because each model's range of utilities is different, the corresponding softmax parameters $\tau$ that produce equal variability will also be different).

To ensure that the pre-determined parameter choices do not inadvertently bias our results, we also fit each model to participant data, allowing us to maximize each model's performance with respect to our task. We report the results of this fitting process in the results section of our main experiment.

\section{Related advances in models of reference}

Before turning to our model evaluations, it is useful to consider recent advances in models of reference production that aim to explain similar phenomena to the ones we do here. In particular, two novel models have been recently proposed to account for the observation that people often produce over-specific referential expressions. The first, the Probabilistic Referential Over-specification (PRO) model, posits a generative model that probabilistically chooses to include or omit adjectives (van Gompel et al., 2019). By adjusting the probability of over-specification for different adjective types, this model successfully captures patterns in referential communication. Nonetheless, PRO does not speak to the psychological causes behind the predicted probability of including or omitting adjectives. Thus, this model can be thought of as capturing the probability that people select adjectives, without capturing the processes that give rise to these probabilities. From this standpoint, our model can be thought of as consistent with the PRO model, with our emphasis being on modeling the cognitive processes that give rise to the probabilities of over-specifying, and how they change as a function of the visual context and their usefulness for visual search.

A second related model, continuous semantics Rational Speech Act, or cs-RSA, has extended Rational Speech Act (RSA) models to include noisy semantics with the goal of explaining referential over-specification (Degen et al., 2019). In classical RSA models, speakers generate referential expressions through recursive social reasoning, considering the likelihood that a listener will recover the intended referent, who in turn, considers why the speaker chose the words that they did (Frank \& Goodman, 2012; Goodman \& Frank, 2016). These models classically use a deterministic association between words and meanings did not directly account for over-specification (i.e., a speaker should not say 'red apple' when 'apple' already reveals the intended target). cs-RSA extends RSA by using a noisy probabilistic association between words and meanings. In doing so, cs-RSA naturally produces a tendency to over-specify, with the goal of reducing noise in the utterance (e.g., cs-RSA might select 'red apple' when a single apple is in view, to avoid the chance that a listener might select the wrong item under the bare description 'apple' due to the noise in the meaning of the word apple).

cs-RSA explains over-specification by manipulating the informativity of words (the first term in Eq. 1; Degen et al. 2019). Our work differs from this approach by focusing instead on the structure of costs (the second term in Eq. 1, and proposing an allocentric structure that estimates effort in visual search. Thus, while our work provides a different route to explain over-specification, it is also not in direct conflict with cs-RSA. It is possible that over-specification is both driven by noisy semantics and by an attempt to provide efficient search means for the listener.

\section{Validating speaker-listener alignment}

Our computational model relies on two critical assumptions: (i) color is more visually salient than material, and (ii) speakers are aware of how this affects visual search. To ensure that these assumptions were reasonable, we began with an experiment that tested for their plausibility by 
employing the same visual materials with both listeners and speakers. Thus, the first model assumption was tested in an eye-tracking task in which listeners had to identify a target in a display of objects following either a color or a material description. If assumption (i) is correct, color descriptions should result in faster target identification than material descriptions. The second model assumption was tested in a description-rating task in which speakers had to rate the same color and material descriptions according to how likely they would be to use them to refer to the target in the display. If assumption (ii) is correct, speakers should rate color descriptions higher than material descriptions, giving higher scores to those descriptions that resulted in faster target identification in the eye-tracking task.

\subsection{Participants}

For the eye-tracking task, 32 participants were recruited from the volunteer pool at the MIT Brain and Cognitive Sciences Department. Participants included students and local residents in the Cambridge area, who were paid for their contribution. All participants reported being native speakers of English and having accurate color discrimination. For the description-rating task, 25 participants were recruited via Amazon's Mechanical Turk crowd-sourcing platform. Recruitment was limited to participants located in the US territory (according to their IP address) and who had a $95 \%$ reliability rate from previous performance on MTurk tasks.

\subsection{Stimuli}

30 displays with pictures of 4 objects were created such that all objects were of different colors and materials. Each display contained a target and a contrast object that was of the same kind as the target, but differed in color and material (e.g., a red leather chair vs a green plastic chair; see Fig. 2 ). To ensure that the color and material of the target and the contrast object were discriminable in the pictures, the materials were pre-tested on an additional 10 participants from the MIT BCS pool.

For the eye-tracking task, each display was paired with two descriptions of the target object, one mentioning its color (e.g., 'The red chair') and another mentioning its material (e.g., 'The leather chair'). The recordings were made by a female native speaker of American English.

The description-rating task employed the same color and material descriptions of the target, but presented both in written under each display. The visual displays were the same as in the eyetracking task, only that the target object appeared inside a grey frame (so that participants would evaluate each description in relation to that object).

\subsection{Procedure}

For the eye-tracking task, displays and descriptions were distributed in two lists using a Latin square design, and participants were evenly allocated to either list. Each trial lasted $3000 \mathrm{~ms}$. In between trials, participants had to click on a cross in the center of the screen. This was done to ensure that the cursor was equidistant from the 4 objects at the start of each trial. Item presentation was individually randomized.

In the description-rating task, participants were asked to rate the color vs material descriptions of each target on a scale from 0-100 according to how likely they would be to use each description in that particular context. Item presentation was individually randomized. 


\subsection{Results}

In the eye-tracking task, net dwell time was calculated separately for the target and the contrast object for the duration of each trial. We ran three linear mixed effects models in which we modeled Adjective Type as a predictor of (1) percentage of net dwell time on the target, (2) percentage of net dwell time on the contrast object and (3) response times (measured from the onset of the description until the participant clicked on an object). In addition to the fixed effect for Adjective Type, the models included random slopes for Adjective Type for subject and item and random intercepts for subject and item (i.e. the maximum random effect structure; see Supplemental Materials for details).

The first model revealed that participants fixated more on the target in the Color Adjective condition than in the Material Adjective condition $(\beta=3.911 ; t=3.423)$, confirming that color is indeed more salient than material. The second model showed that participants fixated more on the contrast object in the Material Adjective condition than in the Color Adjective condition, which suggests that searching by material created more hesitation between the two potential targets (e.g., the leather chair vs the plastic chair) than searching by color (e.g., distinguishing the red chair from the green chair) $(\beta=-7.039 ; t=-5.801)$. These looking patterns are in line with the distinction that our visual search model makes between properties that can be identified from the periphery (such as color) and properties that require directly fixating on the object (such as material). Finally, the third model revealed participants were also significantly faster to click on the target object in the Color condition than in the Material condition $(\beta=-402.37 ; t=-9.669)$. It is important to bear in mind that the visual displays were the same in the two conditions: what changed was the description of the target (e.g., 'The red chair' vs 'The leather chair'), which in turn had an effect on the efficiency of the visual search for the object.

In the description-rating task, participants rated color descriptions almost twice as high as material descriptions (mean ratings: 92 vs $49 ; t(29)=17.666 ; p<.0001$ ). These results support our second assumption: speakers are sensitive to the differential visual salience of color and material properties, preferring to use color descriptions than material descriptions to refer to the targets in our displays.

More importantly for the test of our model assumptions, there was a strong, negative correlation between the average description rating and the average $\mathrm{RT}$ for each color and material description $\left(r=-0.878\left(\mathrm{CI}_{95 \%}:-0.93--0.80\right)\right)$, confirming that speakers preferred to use those descriptions that led listeners to faster target identification. Moreover, the correlation between average rating and average RT remained significant for each adjective type separately (Color: $r=-0.45\left(\mathrm{CI}_{95 \%}\right.$ : $-0.697--0.107)$; Material: $\left.r=-0.54\left(\mathrm{CI}_{95 \%}:-0.754--0.223\right)\right)$.

\section{Explaining referential over-specification}

To evaluate the Incremental Communicative Efficiency (ICE) model's capacity to explain reference production, we began by testing whether it could reproduce known qualitative phenomena in referential over-specification. Specifically, previous work has found that (i) speakers are more likely to use redundant color words in visual displays with more objects (Rubio-Fernandez, 2019; Koolen et al., 2016; Gatt et al., 2017; Paraboni et al., 2007); (ii) this propensity, however, decreases as a function of the number of objects that share the same color as the target referent (Long et al., 2020; Rubio-Fernández, 2016; Rubio-Fernandez, 2019; Koolen et al., 2013); and (iii) in identical visual displays with the same target, English speakers (who position adjectives before nouns) are more likely to use redundant color words relative to Spanish speakers (who position adjectives after nouns; RubioFernández 2016; Rubio-Fernandez 2019; Rubio-Fernandez et al. 2020). These phenomena show that people do not have a simple bias towards producing color words, but that this preference is nuanced, 
a)

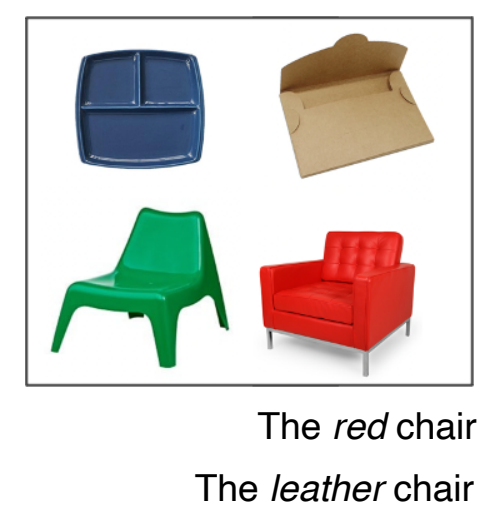

d)

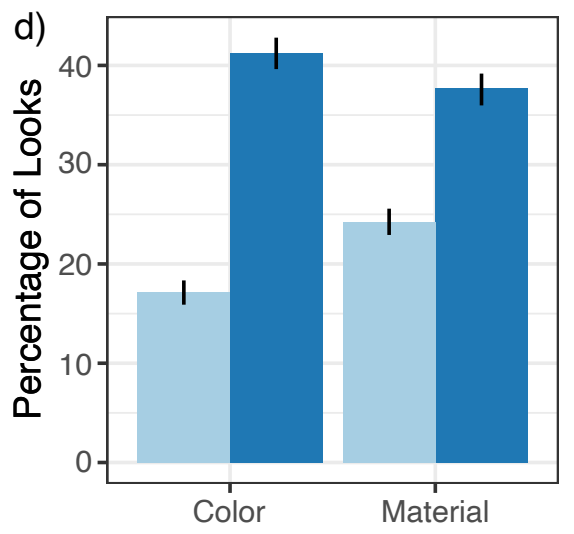

Contrast
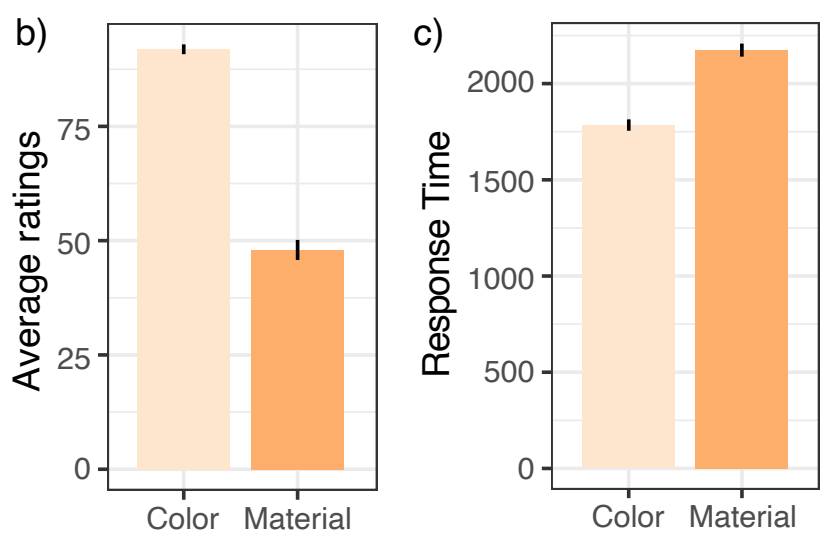

e)

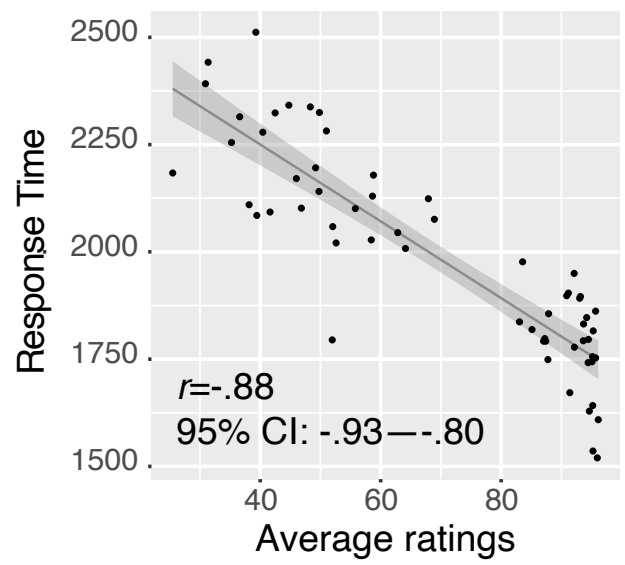

Figure 2: Sample trial and results from the test of our model assumptions. a) Example trial. The four objects in the sample display have been enlarged for illustrative purposes. Each trial had a target object (the red leather chair, in this case) with a competitor (the green plastic chair, in this case). b) Average ratings for the two descriptions of each target (i.e. how likely participants would be to use the color or the material description to refer to the target) in the description-rating task. Participants showed a reliable preference for color descriptions over material descriptions. c) Average response time as a function of adjective in the instructions in the visual search task. Participants were significantly faster to click on the correct target when they heard a color adjective (e.g., 'the red chair') relative to a material adjective (e.g., 'the leather chair'). d) Percentage of fixations on the Target and the Contrast object (e.g., the red leather chair vs the green plastic chair) in the visual search task. d) Average rating in the description-rating task ( $\mathrm{x}$ axis) against response time ( $\mathrm{y}$ axis), showing that descriptions rated as more likely to be produced were the ones that led listeners to identify the referent faster. Error bars indicate $95 \%$ bootstrapped confidence intervals. 
and shaped by display density, color gamut, and word order - as the incremental efficiency theory predicts.

To evaluate if our model could reproduce these effects, we considered a simplified version of our efficient search model that only considered bare definite descriptions (e.g., 'the star') and colormodified descriptions (e.g., 'the blue star'), as these phenomena have been primarily established with paradigms where material information is not available (e.g., by using simple geometrical shapes of different colors). Throughout, we used the pre-set model parameters and did not change them as a function of the phenomena or dataset that we evaluate.

\subsection{Redundancy as a function of display density}

To test the first effect - speakers are more likely to use redundant color words in visual displays with more objects - we created visual displays that ranged from 3 to 16 objects, all with a unique target of a unique color (e.g., a single blue star in an array of geometrical shapes, none of which were blue or stars). Because in all cases, the bare description is sufficiently informative, the alternative brevity model predicts that speakers should never use color words, regardless of the objects in the display.

Figure 3a shows the results from this analysis. Like people, our model's preference for redundant color words increases as a function of the number of objects in the scene.

\subsection{Redundancy as a function of color variation}

We next tested whether our model's propensity to use redundant color words decreased when all objects were the same color. To test this possibility, we created arrays of nine objects, all with a unique target (e.g., a single star among a set of geometrical shapes). We then manipulated the display's monochromaticity, ranging from $1 / 9$ (the target has a unique color) to $9 / 9$ (all objects share the same color).

Figure $3 \mathrm{~b}$ shows the results from this analysis. Once again, our model was able to reproduce the empirical pattern where monochromaticity reduces people's propensity to use redundant color words. Note that, once again, because the target could be uniquely specified by the noun, a brevitybased model, like our alternative model, would always favor the bare description, independent of the distribution of colors in the display.

\subsection{Cross-linguistic variation}

We next sought to test whether our model could reproduce cross-linguistic phenomena. When describing the same target in equivalent displays, English speakers - who position adjectives prenominally - are more likely to use redundant color words relative to Spanish speakers - who position adjectives post-nominally. To test for this possibility, we created arrays of 3 to 16 objects, all of them with a unique target of a unique color (e.g., as with the first analysis, a single blue star in an array of geometrical shapes, none of which were blue or stars). We then ran our original model along with a modification of our model that positions adjectives post-nominally.

Figure 3c shows how our model reproduced this cross-linguistic effect. Our model's preference for redundant color words increased as a function of objects in the display with both language structures. However, the preference for redundant color words was higher when adjectives were positioned pre-nominally (as in English) relative to post-nominally (as in Spanish), as reported empirically in Rubio-Fernandez et al. (2020). 

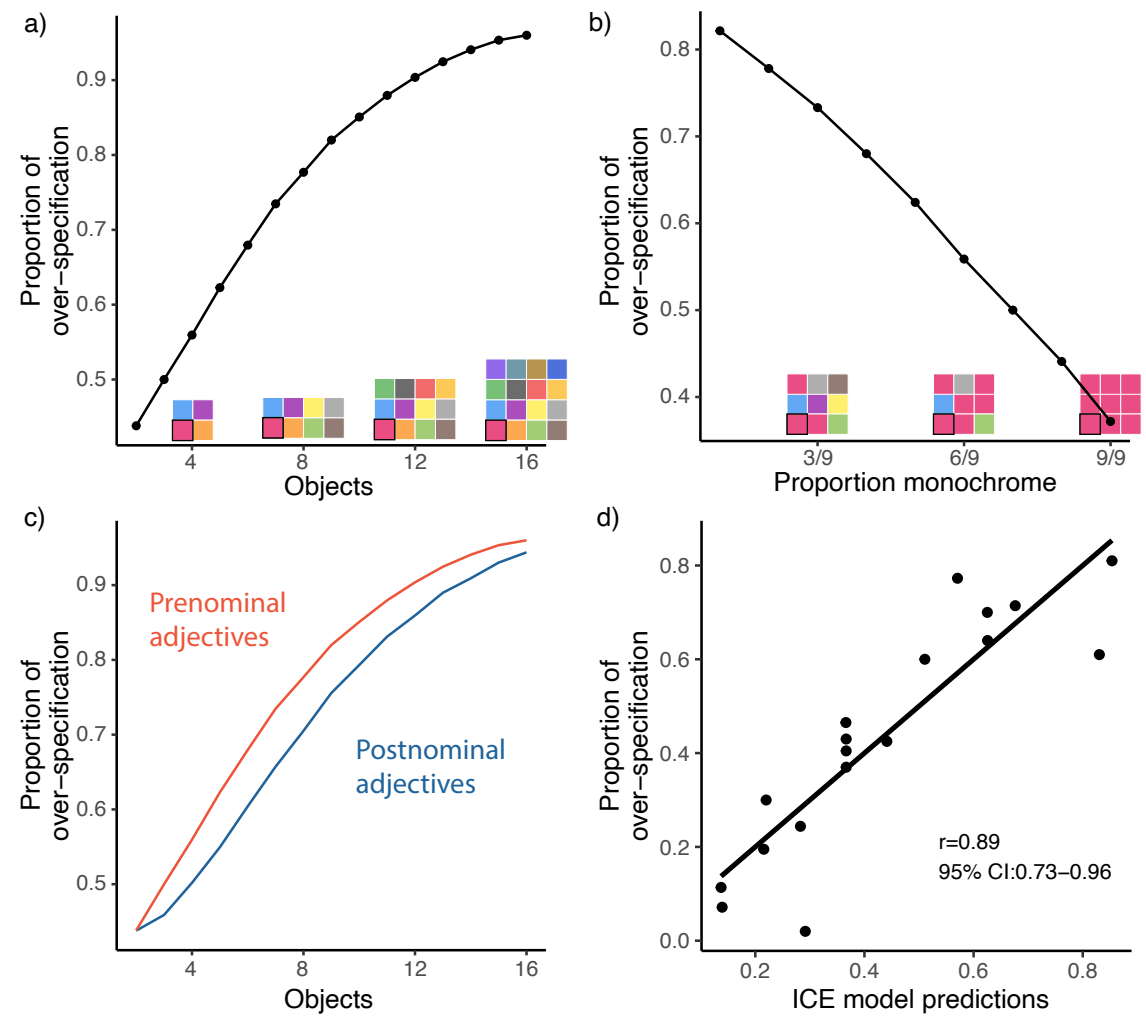

Figure 3: Model simulations and fits to documented effects on redundant color use. a) Number of objects in a visual scene ( $\mathrm{x}$ axis) against model probability of producing redundant color words (y axis). Consistent with (RubioFernandez, 2019), our model is more likely to use redundant color words in denser (polychromatic) visual scenes. b) Proportion of objects of the same color in a scene with nine objects (x axis) against model probability of producing redundant color words (y axis). Consistent with (Long et al., 2020; Rubio-Fernandez, under review), our model decreases its propensity to use color words as a function of monochromaticity. c) Number of objects in a visual scene ( $\mathrm{x}$ axis) against model probability of producing redundant color words ( $\mathrm{y}$ axis) based on adjective positioning. Consistent with (Rubio-Fernandez et al., 2020), our model is more likely to use redundant color words when it positions adjectives prenominally (as in English) relative to postnominally (as in Spanish). d) model predictions (x axis) against empirical data (y axis) from previous studies.

\subsection{Quantitative model fit to previous data}

Having found evidence that our model could reproduce the qualitative phenomena behind the incremental efficiency theory, we then tested whether it could quantitatively fit the data reported in these papers. To achieve this, we combined the following four datasets consisting of production data elicited in interactive laboratory tasks.

The first dataset consists of polychrome displays of increasing density (2, 4, and 8 shapes) from Rubio-Fernandez (2019) showing that speakers increase redundant color use in denser displays. The second dataset consists of two types of 4-shape displays, one monochrome (all objects of the same color) and one polychrome (all objects of different colors), used to show a reduced used of redundant color words in monochrome displays, from Long et al. (2020).

The third dataset shows the same effect, but using a graded set of conditions where all displays had nine objects and the proportion of monochrome objects varied from one to nine, from (RubioFernandez, under review). Finally, our fourth dataset consisted of four- and sixteen-item polychrome 
displays with production data from Spanish and English speakers, from Rubio-Fernandez et al. (2020).

These four datasets capture the three phenomena in color over-specification that our model explains qualitatively. Because each dataset consists of only a few key contrasts, we combined all data (for a total of $n=18$ conditions tested) and compared averaged judgments against our model, using once again our pre-set model parameters.

People's propensity to use redundant color words is affected by a multitude of factors, including color discriminability (Viethen et al., 2017), perceptual grouping (Koolen, 2019), and color typicality (Sedivy, 2003; Rubio-Fernández, 2016; Westerbeek et al., 2015), among others. We therefore expected our model to capture quantitative graded phenomena across conditions, but not necessarily the baseline levels of redundancy, which are likely influenced by additional factors. Thus, to make our results easier to interpret, we used a linear regression to predict judgments as a function of model predictions. Note that correlations are invariant to linear transformations, and the application of a linear regression therefore does not affect any of our statistical results, and instead, it only helps show how quantitative changes in the model relate to quantitative changes in people's propensity to over-specify.

Figure $3 \mathrm{~d}$ shows the results from this analysis, with model predictions on the $\mathrm{x}$ axis and overspecification in these papers on the y axis. Our model showed a correlation of $r=0.89\left(\mathrm{CI}_{95 \%}\right.$ : $0.73-0.96)$. This high correlation shows strong evidence for the ICE model, given that this data was obtained from previous results with varying paradigms and that we did not have to fit separate parameters to each dataset. Because, in all of these datasets, the target can always be uniquely specified with the noun alone, the alternative Brevity model treats all of these events as equivalent and produces no variance throughout (and thus making it impossible to compute a correlation).

\section{Testing the Incremental Communicative Efficiency (ICE) model}

Our results so far show that the ICE model explains patterns of reference production and overspecification in interactive laboratory tasks. Nonetheless, production tasks can hide away people's intuitions about expressions that they choose not to produce. Thus, to evaluate our model in a more holistic way, we also designed a graded acceptability task in which we asked participants to rate how natural different descriptions sounded, allowing us to evaluate our model not only based on its preferred expression, but also on the full distribution of expressions that it produces.

\subsection{Methods}

\subsubsection{Participants.}

41 participants (mean age $=34.2$ ) were recruited through Amazon's Mechanical Turk framework. Two additional participants were recruited but excluded in the study because they failed at least one catch trial (see Results).

\subsubsection{Stimuli.}

Stimuli consisted of 30 critical trials, each consisting of a grids of objects like those in Fig.4. Objects came in 9 colors: black, blue, brown, green, orange, pink, purple, red and yellow; and in 9 materials: cardboard, ceramic, glass, leather, metal, paper, plastic, wood, and wool. The target object appeared inside a grey frame and its position was counterbalanced across trials. The 30 trials consisted of three different density levels: three objects ( 8 trials), six objects (10 trials), and nine objects (12 trials). In 3 of these trials (one of each density), the target object was of a unique category, color and material, making modification unnecessary (e.g., the minimal description 'the 


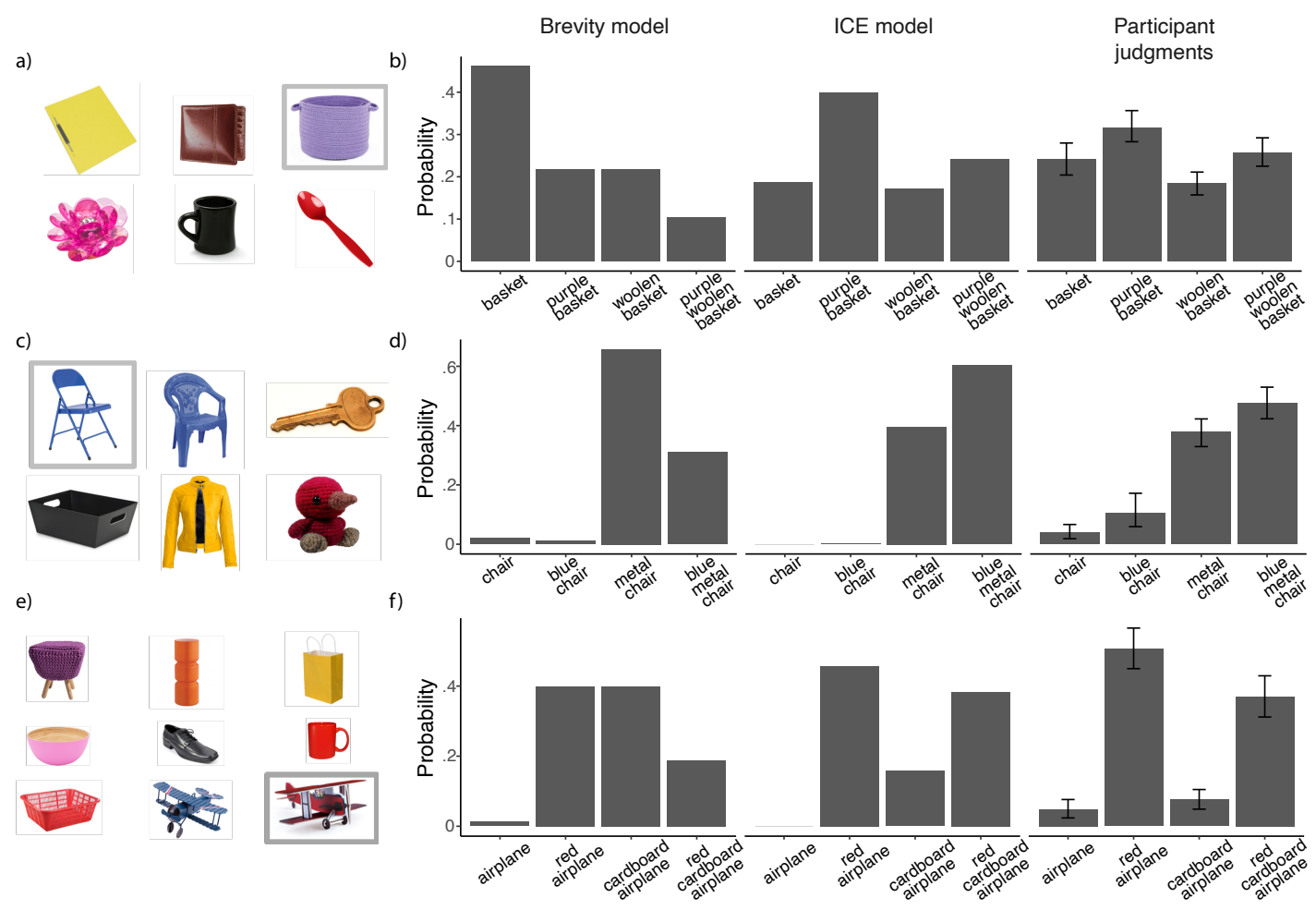

Figure 4: Example trials from our main experiment along with model predictions and participant judgments. a) Display where the target - the basket - can be identified without any modifiers. b) The alternative Brevity model favors the shortest utterance ('the basket') and decays as a function of utterance length. By contrast, the ICE model, like participants, dis-prefer the shortest utterance (despite it being sufficiently informative) and instead introduce a redundant color word. c) Trial where the target must be disambiguated by material rather than color. d) The alternative Brevity model discards the color, while the ICE model and participants use both color and material. e) A situation where either color and material can disambiguate the target. f) The alternative Brevity model favors expressions with a single modifier. The ICE model and participants prefer utterances that include color.

basket' would be sufficiently informative). The remaining 27 trials included a contrast object of the same category as the target (e.g., a second chair) and varied depending on whether the contrast object was of a different color than the target ( 6 trials, 2 of each density); a different material ( 6 trials, 2 of each density), or differed along both dimensions (15 trials, 3 with 3 objects, 5 with 6 objects and 7 with 9 objects). In order to vary the relative efficiency of color and material adjectives for visual search, one or more objects in the display acted as 'competitors' and were the same color as the target and the contrast object ( 1 color competitor in 6 trials, 2 of each density; 2 color competitors in 2 trials, 1 with 6 objects and 1 with 9 objects; 3 color competitors in 1 trial with 9 objects) or the same material as the target and the contrast object (1 material competitor in 6 trials, 2 of each density; 2 material competitors in 2 trials, 1 with 6 objects and 1 with 9 objects; 3 color competitors in 1 trial with 9 objects). See Supplemental Materials for full stimuli. In addition to the 30 critical trials, we included four catch trials, designed to ensure that participants were paying attention. In each of these trials, only one of the four descriptions matched the target, thus allowing us to ensure that participants were reading the expressions when rating them. 


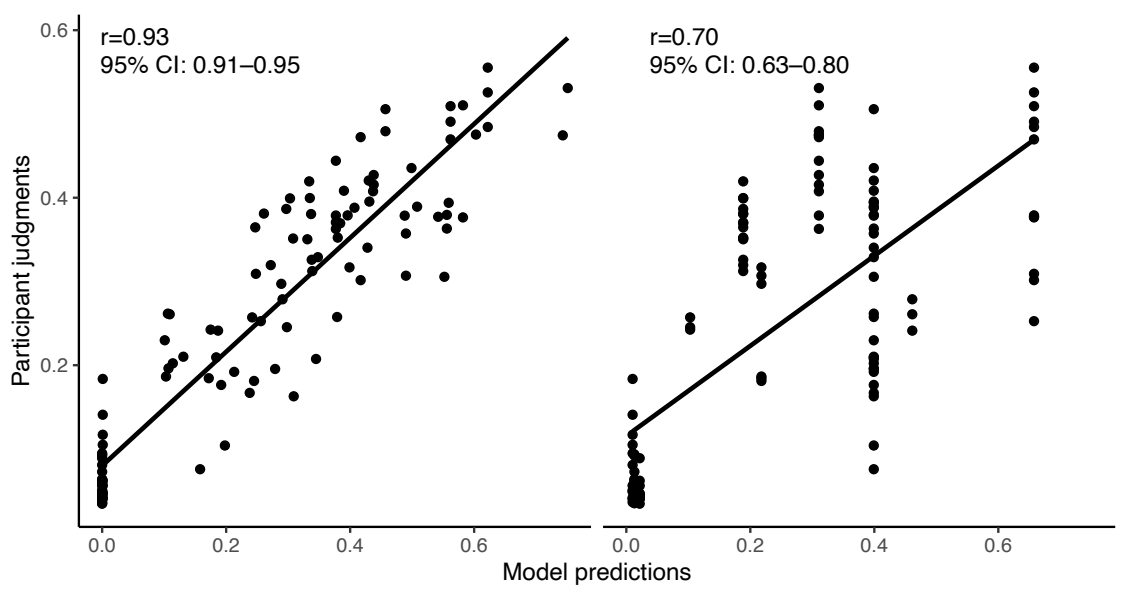

Figure 5: Experiment results. Each dot represents a referential expression (four expressions per trial). The x-axis shows the model predictions and the y-axis shows average participant judgments. Diagonal black lines show best linear fit. First scatterplot shows the ICE model and second scatterplot shows the alternative Brevity model.

\subsubsection{Procedure.}

Participants read a brief tutorial and then completed all trials in a randomized order. In each of the test trials, participants rated on a scale from 0-100 how likely they were to refer to the target in the display by using each of four descriptions ( 0 described as 'Very unlikely', 50 as 'Somewhat likely' and 100 as 'Very likely'), and the sliding button appeared at 50. Descriptions included no modification, a color adjective, a material adjective or both types of adjectives. The order of these descriptions was randomized individually across trials.

The four catch trials were identical with the difference that only one of the descriptions matched the target object, and the other 3 described objects that were not present in the display.

\subsubsection{Results.}

Each participant's judgments were normalized within trials so that they added up to 1. Participants who did not place at least $80 \%$ of the probability on the correct description on any of the four catch trials was excluded from the study $(n=2)$. Judgments were then averaged across all participants. Overall, our main (ICE) model showed a correlation of $r=0.93\left(\mathrm{CI}_{95 \%}: 0.91-0.95\right)$, while the alternative (brevity) model showed a lower correlation of $r=0.70\left(\mathrm{CI}_{95 \%}: 0.63-0.80\right)$. Moreover, our ICE model showed a significantly higher correlation relative to the alternative model $\left(\Delta r=0.22 ; \mathrm{CI}_{95 \%}: 0.14-0.29\right)$.

As noted in the computational framework, the parameters for our ICE model (fixation rate $=1$ with $\tau=2$ ) were set prior to data collection and the alternative Brevity model's parameters were selected so as to make the two models comparable (cost $=0.09$ with $\tau=0.12$; see Computational Framework for explanation). To test whether our results were affected by this initial choice, we next fit each model's parameter to participant data to maximize their performance (as determined by the correlation; see Supplemental Materials for details). Fitting the Brevity model's parameters to participant data (cost=0.01 with $\tau=0.31$ ) increased the model's correlation to $r=0.88\left(\mathrm{CI}_{95 \%}\right.$ : $0.84-0.93)$. While this correlation was substantially higher than the one we obtained with the pre-set parameters $(\Delta=.18$ increase), it was still reliably lower than the correlation from our ICE 
model with pre-set parameters $\left(\Delta r=0.04 ; \mathrm{CI}_{95 \%}: 0.006-0.08\right)$. Thus, the ICE model with pre-set parameters outperformed even a Brevity model fit to participant judgments. Similarly, the parameters that best fit our model to participant data (fixation rate $=1$ with $\tau=2.9$ ) increased the correlation to $0.94\left(\mathrm{CI}_{95 \%}: 0.92-0.96\right)$, which was also reliably higher than the best Brevity model fit $\left(\Delta r=0.06 ; \mathrm{CI}_{95 \%}: 0.02-0.09\right)$.

Figure 4 shows three example trials that illustrate the differences between the two models (using the pre-set model fits). The event in Figure 4a consists of six objects with a target basket. Because there is only one basket, the Brevity model selects 'basket' as the best utterance (as it is sufficiently informative, and carries the minimal cost), followed by 'purple basket' and 'woolen basket', as they both use one unnecessary word, and ending with 'purple woolen basket' which uses two unnecessary words (Fig. 4b). By contrast, the ICE model mimics participant judgments, favoring 'purple basket' and 'purple woolen basket', because locating a purple object is easier than finding a basket (Fig. 4b). Fig. 4c shows a situation where mentioning the object's material is necessary. Consequently, the Brevity model prefers 'metal chair' over 'blue metal chair'. Our ICE model and participants, however, prefer 'blue metal chair' over 'metal chair' (Fig. 4d), which help the listener first identify the blue objects, and find the metal one between those two (rather than relative to the entire visual scene). Finally, Fig. 4d shows a case where the referent can be identified through color or material. The Brevity model assigns equal probability to 'red airplane' and 'cardboard airplane' and a lower probability to 'red cardboard airplane'. Our ICE model and participants instead assign the highest probability to 'red airplane', followed by 'read cardboard airplane', and disprefer 'cardboard airplane', despite it being sufficiently informative.

The Brevity model with the best parameter fits also reveals how this model fails to explain participant judgments. The fitting process selected the lowest possible word cost available in our search space (0.01; see Supplemental Materials for detail), suggesting that the Brevity model performed better when the egocentric word cost was minimized, further suggesting that word costs had minimal explanatory power.

\section{Discussion}

Here we presented a computational model of the incremental efficiency theory, which posits that speakers generate referential expressions by considering listeners' visual search. Our model was built on the assumptions that speakers expect listeners to process words incrementally, and that people can identify color more easily relative to materials and categories. Related research has shown that listeners indeed process words incrementally (Eberhard et al., 1995; Rubio-Fernandez et al., 2020), and that they are often quicker to identify a referent when they are provided with a (technically redundant) color word, despite the utterance being longer (Sonnenschein \& Whitehurst, 1982; Mangold \& Pobel, 1988; Paraboni et al., 2007; Paraboni \& Van Deemter, 2014; Arts et al., 2011; Tourtouri et al., 2019; Rubio-Fernandez, under review). Moreover, our speaker-listener alignment study (section 6) revealed a direct link between these listener effects and speaker linguistic preferences: descriptions rated as more appropriate for a visual context strongly correlated with listener reaction time when processing that same description. Our work thus suggests that the principles by which speakers generate referential expressions are aligned with how listeners process these expressions and search for the corresponding referents.

Our computational model diverges from the standard interpretation of the Gricean Maxim of Quantity (Grice et al., 1975), according to which communicative efficiency is equated to brevity (Sedivy, 2003; Pogue et al., 2016; Engelhardt et al., 2006; Davies \& Katsos, 2013; Engelhardt et al., 2011). Instead, our model does not treat words as costly and assumes that speakers are motivated to help their listeners identify the intended referent as efficiently as possible. In doing so, our 
model captured nuanced patterns of reference that the Brevity account could not explain, and it spontaneously gave rise to empirical phenomena that, at first sight, might appear to challenge the Gricean Maxim of Quantity. Specifically, our model reproduced people's propensity to use redundant color words as a function of display density, the reduction of redundant color words in monochrome displays, and the cross-linguistic variation observed as a function of adjective positioning (Section 7). Critically, our framework explains all of these effects as forms of efficient communication. Our model's formalization of referential efficiency is therefore in line with Grice's view of communication as a rational and cooperative activity.

Our findings directly speak to a much debated question in the pragmatics literature: whether, and to what extent, speakers tailor their utterances to their interlocutors (Clark \& Murphy, 1982; Horton \& Keysar, 1996; Clark \& Krych, 2004). This is known as audience design. Referential communication studies have shown that speakers adjust their referential expressions to the needs of their interlocutors, distinguishing experts from novices, for example (Heller et al., 2012; Isaacs \& Clark, 1987). Recent work has also found that computational frameworks centered around audience design - where utterances aim to maximize the chance that listeners recover the intended messagesuccessfully capture a wide range of linguistic phenomena, from scalar implicature to hyperbole (Goodman \& Frank, 2016). Our work advances this view, showing the nuance in audience design: Speakers produce utterances tailored to help listeners efficiently recover the intended message in real-time, showing sensitivity to the visual context and to their interlocutors' visual search.

\subsection{Implications of the structure and nature of referential costs}

Most directly, our results show that an allocentric cost function explains how speakers produce referential expressions. Additionally, we also found that a model with an egocentric brevity cost function failed to capture both qualitative and quantitative patterns of reference. Even when the parameters of this alternative Brevity model were fit to participant data, the best fit under-performed relative to our Incremental Communicative Efficiency (ICE) model. Interestingly, the best Brevity model obtained through parameter fitting was the one that used the smallest possible production cost available in our parameter space. These results suggest that utterance-length penalization had minimal explanatory power in our task, to the point that the model performed better by giving the lowest possible weight. These results are consistent with related work that has also found that egocentric production costs do not confer significant benefits to reference models (Degen et al., 2019). While this finding might seem surprising in the context of the standard interpretation of the Gricean Maxim of Quantity, we believe these results are intuitive - in everyday conversations, longer utterances rarely feel more costly, and people often say more than what would be considered necessary by a rigid brevity analysis.

At the same time, our results do not imply that reference is guided purely by allocentric costs. Related work suggests that linguistic preferences in speakers are also shaped by egocentric costs, such as the effort associated with preempting an ambiguity (Ferreira et al., 2005) or adopting the listener's visual perspective (Nadig \& Sedivy, 2002). Thus, our work adds to these previous findings by showing that referential costs are partially allocentric, guided by a sensitivity to listeners' visual search. However, in more complex situations, speakers' costs must integrate both egocentric and allocentric components. While numerous psycholinguistic studies have investigated audience design, the space of costs that shape reference production remain poorly understood (for a review of so-called 'speakerinternal' and 'addressee-oriented processes' in reference production, see Arnold 2008). Nonetheless, our work shows that the space of these costs is conceptually rich, and must include allocentric components that depend on a consideration of our listeners, and not only on an expectation of brevity. 
Our work also reveals an interesting conceptual distinction between production and selection costs. That is, some cost functions represent which referential expressions come to mind, while other cost functions capture information that speakers compute when deciding which expression to produce. Consider, for instance, a speaker who can describe an object as 'the sofa' or 'the chaise'. In a situation like this, the speaker might be more likely to select the first expression because the word 'sofa' is easier to retrieve from memory than the word 'chaise'. This preference can be captured by a production cost that represents the difficulty of retrieving different words from memory (and is therefore not a cost that the speaker calculates). Suppose, however, that the speaker is a furniture salesman, who has little problem retrieving both expressions from memory. In this case, the speaker might nonetheless be more likely to use the expression 'the sofa' because the listener will have an easier time processing it, but they might also select 'the chaise' if their costumer seems to be looking for something exclusive. These different preferences can be captured by a selection cost that represents information that the speaker is calculating about different expressions when deciding which one to use.

This distinction suggests that cost functions in reference can both represent the difficulty of generating different expressions (production costs) and the value that speakers assign to expressions that they can easily generate (selection costs). Intuitively, allocentric costs are typically of the selection type (they are estimated to decide what a listener will find useful), while egocentric costs are typically of the production type (they are forces that shape what comes to speakers' minds). While similar distinctions have been made in psycholinguistic studies of audience design (see Arnold 2008), to the best of our knowledge, it remains an open question the extent to which different types of costs are production-based or selection-based.

\subsection{Relationship between referential communication and social cognition}

Our model builds referential expressions by considering listeners' visual search. An open question is the extent to which this process involves active perspective taking. Research in Theory of Mind has long argued that it is critical to distinguish our own beliefs and desires from those of others, because these have great variability across agents (Repacholi \& Gopnik, 1997; Wimmer \& Perner, 1983). Visual systems, however, are largely homogeneous. And while agents often differ in what they can see from on their physical position in space, the principles of what we find visually salient (such as color) are the same for everyone. Thus, it is possible that speakers rely on their own visual system to estimate the listener's difficulty in identifying the referent.

Even if speakers produce efficient referential expressions by relying on their own visual system, this process would still require some amount of perspective taking. At a minimum, the speaker needs to be aware that they are sharing a physical space with their listener (a situation known as co-presence; Clark \& Marshall 1981) and can therefore rely on mutual salience (Rubio-Fernandez, 2019). As obvious as this might seem, it is important to note that this assumption is not a default in human communication: particularly these days with the ongoing pandemic, we spend more time communicating with others over the phone or in Zoom meetings where we cannot rely on mutual salience, than in situations of co-presence. Therefore, monitoring whether we can or we cannot rely on mutual salience is a form of perspective taking that is necessary for successful, efficient referential communication.

Another form of perspective taking that underlies efficient referential communication is the speaker's prior experience as a listener: having previously processed different types of adjectives as listeners, speakers have first-hand information about which visual properties are more efficient for visual search (Rubio-Fernandez, under review). Thus, an efficient speaker in a situation of copresence can safely rely on their prior communicative and perceptual experience, without necessarily having to mentally simulate the listener's perspective (for social neuroscience studies using simulated 
perspective taking, see (Ruby \& Decety, 2001; Blanke et al., 2005; David et al., 2006; Bio et al., 2018).

\subsection{Model limitations}

The goal of our paper was to present a formal computational framework for the incremental efficiency theory that enabled us to clarify how allocentric costs might be computed, and to test its explanatory power in a quantitative manner. These results open new questions that the model presented here is not yet equipped to answer. In particular, our model has two limitations.

First, our model used a relatively coarse distinction between color adjectives and material adjectives, but we did not distinguish between different types of colors and materials, nor their salience in different scenes. Thus, for instance, in our model, saying the 'red triangle' is equally useful when the target is surrounded by blue objects, than when it is surrounded by orange objects. Intuitively, however, identifying the red object is easier in the first situation relative to the second. To account for this, it is critical to develop a more nuanced model of visual search that estimates visual salience based on the entire context (Itti et al., 1998; Itti \& Koch, 2012; Coco \& Keller, 2001).

A relative limitation is that our model focused only on color and material adjectives, but people can use a variety of additional adjectives to refer to a target, including the size, texture, shape, and even purpose. While our framework can be easily extended to handle additional adjective types, an appropriate model would require knowledge about the correlation between objects and their features. For instance, when referring to a banana, its color might be useful for visual search, but a speaker might still choose to not say 'yellow banana' because the noun 'banana' already reveals that the object is yellow (Sedivy, 2003). Implementing this extension would also give our model the capacity to favor unusual adjectives. In the same example, if the banana were blue, such speaker model would correctly anticipate that 'banana' would trigger a yellow-based search in the listener, and thus would produce 'blue banana' to avoid this (Westerbeek et al., 2015; Rubio-Fernández, 2016; Degen et al., 2020). Expanding our framework to handle multiple adjective types using the underlying correlational structure is a direction that we hope to explore in future work.

\subsection{Future directions}

Our computational model implemented the incremental efficiency theory in the context of referential communication about objects present in a scene. This enabled us to test the idea that speakers select adjectives through an allocentric cost estimation based on listeners' expected visual search.

Nonetheless, the incremental efficiency theory makes predictions about a broader class of communicative interactions. For instance, when discussing events in common ground, or when referring to abstract ideas, listeners' visual search is no longer the guiding metric of efficiency for listener understanding. In cases like these, incremental efficiency predicts that speakers should structure their message so as to include properties that help the listener interpret the message quickly as they process language incrementally. Thus, speakers may be more inclined to begin with memorable properties over non-memorable ones. For instance, if 'the tall man' and 'the mean man' refer to the same person, the first expression might be more efficient when helping the listener identify the man in a crowd, while the second expression might be more efficient when asking the listener to recall someone they previously encountered.

While these broader predictions of the incremental efficiency theory remain to be tested empirically, the Incremental Communicative Efficiency framework provides a first step towards modeling these types of inferences. Under our framework, this would require using an allocentric cost function that models out intuitive theory of 'the mind's eye' and how it brings information in memory to bear. This is a direction that we hope to explore in future work, helping further test the scope of the 
incremental efficiency theory in a computational manner, and to reveal the extent to which people can use rich intuitive models to compute allocentric costs in communicative interactions.

\subsection{Conclusions}

Overall, our work advances the view that communication is best understood as a social activity where interlocutors share a joint goal to transmit their intended messages efficiently. Our work shows that, even in the simplest linguistic activities, such as deciding what to call an object, speakers construct reference with their listeners in mind, attending not only to how much information their utterances contain, but also to how their listeners will extract the meaning that is conveyed. Our results, in turn, advance an interpretation of the Gricean Maxim of Quantity where efficiency is not quantified through an egocentric bias to be brief, but through our nuanced understanding of other people's minds.

\section{Acknowledgments}

We thank Maddie Long for help collecting data, and Vishakha Shukla and Vrinda Bhatia for help coding the data. We thank Sammy Floyd for useful comments.

\section{References}

Arnold, J. (2008). Reference production: Production-internal and addressee-oriented processes. Language and cognitive processes, 23, 495-527.

Arts, A., Maes, A., Noordman, L., \& Jansen, C. (2011). Overspecification facilitates object identification. Journal of Pragmatics, 43, 361-374.

Bio, B. J., Webb, T. W., \& Graziano, M. S. A. (2018). Projecting one's own spatial bias onto others during a theory-of-mind task. Proceedings of the National Academy of Sciences, 115, E1684-E1689.

Blanke, O., Mohr, C., Michel, C. M., Pascual-Leone, A., Brugger, P., Seeck, M., \& Thut, G. (2005). Linking out-of-body experience and self processing to mental own-body imagery at the temporoparietal junction. Journal of Neuroscience, 25, 550-557.

Bridgers, S., Jara-Ettinger, J., \& Gweon, H. (2020). Young children consider the expected utility of others' learning to decide what to teach. Nature Human Behaviour, 4, 144-152.

Clark, H. H., \& Krych, M. A. (2004). Speaking while monitoring addressees for understanding. Journal of memory and language, 50, 62-81.

Clark, H. H., \& Marshall, C. R. (1981). Definite knowledge and mutual knowledge, .

Clark, H. H., \& Murphy, G. L. (1982). Audience design in meaning and reference. In Advances in psychology (pp. 287-299). Elsevier volume 9.

Coco, M. I., \& Keller, F. (2001). Scan patterns predict sentence production in the cross-modal processing of visual scenes. Cognitive Science, 2, 1204-1223.

Cohn-Gordon, R., Goodman, N. D., \& Potts, C. (2018). An incremental iterated response model of pragmatics. arXiv preprint arXiv:1810.00367, . 
David, N., Bewernick, B. H., Cohen, M. X., Newen, A., Lux, S., Fink, G. R., \& Vogeley, K. (2006). Neural representations of self versus other: Visual-spatial perspective taking and agency in a virtual ball-tossing game. Journal of Cognitive Neuroscience, 18, 898-910.

Davies, C., \& Katsos, N. (2013). Are speakers and listeners 'only moderately gricean'? an empirical response to engelhardt et al.(2006). Journal of Pragmatics, 49, 78-106.

van Deemter, K., Gatt, A., van Gompel, R. P., \& Krahmer, E. (2012). Toward a computational psycholinguistics of reference production. Topics in Cognitive Science, 4, 166-183.

Degen, J., Hawkins, R. D., Graf, C., Kreiss, E., \& Goodman, N. D. (2020). When redundancy is useful: A bayesian approach to "overinformative" referring expressions. Psychological Review, 127, 591-621.

Degen, J., Hawkins, R. X., Graf, C., Kreiss, E., \& Goodman, N. D. (2019). When redundancy is rational: A bayesian approach to'overinformative'referring expressions. arXiv preprint arXiv:1903.08237, .

Dryer, M. S. et al. (2005). Order of adjective and noun. World atlas of language structures, (pp. 353-357).

Eberhard, K. M., Spivey-Knowlton, M. J., Sedivy, J. C., \& Tanenhaus, M. K. (1995). Eye movements as a window into real-time spoken language comprehension in natural contexts. Journal of psycholinguistic research, 24.

Engelhardt, P. E., Bailey, K., \& Ferreira, F. (2006). Do speakers and listeners observe the gricean maxim of quantity? Journal of Memory and Language, .

Engelhardt, P. E., Demiral, Ş. B., \& Ferreira, F. (2011). Over-specified referring expressions impair comprehension: An erp study. Brain and cognition, 77, 304-314.

Fedzechkina, M., \& Jaeger, T. F. (2020). Production efficiency can cause grammatical change: Learners deviate from the input to better balance efficiency against robust message transmission. Cognition, 196.

Ferreira, V. S., Slevc, L. R., \& Rogers, E. S. (2005). How do speakers avoid ambiguous linguistic expressions? Cognition, 96, 263-284.

Frank, M. C., \& Goodman, N. D. (2012). Predicting pragmatic reasoning in language games. Science, 336, 998-998.

Gatt, A., Krahmer, E., van Deemter, K., \& van Gompel, R. P. (2014). Models and empirical data for the production of referring expressions. Cognitive Science, 29, 899-911.

Gatt, A., Krahmer, E., Van Deemter, K., \& van Gompel, R. (2017). Reference production as search: The impact of domain size on the production of distinguishing descriptions. Cognitive Science, 41, 1457-1492.

van Gompel, R. P., van Deemter, K., Gatt, A., Snoeren, R., \& Krahmer, E. J. (2019). Conceptualization in reference production: Probabilistic modeling and experimental testing. Psychological Review, 126, 345-373.

Goodman, N. D., \& Frank, M. C. (2016). Pragmatic language interpretation as probabilistic inference. Trends in Cognitive Sciences, 20, 818-829. 
Grice, H. P., Cole, P., Morgan, J. et al. (1975). Logic and conversation. 1975, (pp. 41-58).

Heller, D., Gorman, K. S., \& Tanenhaus, M. K. (2012). To name or to describe: Shared knowledge affects referential form. Topics in Cognitive Science, 4, 290-305.

Horton, W. S., \& Keysar, B. (1996). When do speakers take into account common ground? Cognition, 59, 91-117.

Isaacs, E. A., \& Clark, H. H. (1987). References in conversation between experts and novices. Journal of experimental psychology: general, 116, 26-37.

Itti, L., \& Koch, C. (2012). Computational modelling of visual attention. Nature reviews neuroscience, 36, 194-203.

Itti, L., Koch, C., \& Niebur, E. (1998). A model of saliency-based visual attention for rapid scene analysis. IEEE Transactions on pattern analysis and machine intelligence, 20, 1254-1259.

Jara-Ettinger, J., Floyd, S., Huey, H., Tenenbaum, J. B., \& Schulz, L. E. (2019). Social pragmatics: Preschoolers rely on commonsense psychology to resolve referential underspecification. Child development, .

Jara-Ettinger, J., Gweon, H., Schulz, L. E., \& Tenenbaum, J. B. (2016). The naïve utility calculus: Computational principles underlying commonsense psychology. Trends in cognitive sciences, 20, 589-604.

Jara-Ettinger, J., Schulz, L. E., \& Tenenbaum, J. B. (2020). The naive utility calculus as a rational, quantitative foundation for action understanding. Cognitive Psychology, .

Kao, J. T., Wu, J. Y., Bergen, L., \& Goodman, N. D. (2014). Nonliteral understanding of number words. Proceedings of the National Academy of Sciences, 111, 12002-12007.

Koolen, R. (2019). On visually-grounded reference production: Testing the effects of perceptual grouping and 2d/3d presentation mode. Frontiers in Psychology, 10.

Koolen, R., Goudbeek, M., \& Krahmer, E. (2013). The effect of scene variation on the redundant use of colour in definite reference. Cognitive Science, 37, 395-411.

Koolen, R., Krahmer, E., \& Swerts, M. (2016). How distractor objects trigger referential overspecification: Testing the effects of visual clutter and distance. Cognitive Science, 40, 1607-1647.

Krahmer, E., \& van Deemter, K. (2012). Computational generation of referring expressions: A survey. Computational Linguistics, 38, 173-218.

Krahmer, E., Erk, S. v., \& Verleg, A. (2003). Graph-based generation of referring expressions. Computational Linguistics, 29, 53-72.

Liu, S., Ullman, T. D., Tenenbaum, J. B., \& Spelke, E. S. (2017). Ten-month-old infants infer the value of goals from the costs of actions. Science, 358, 1038-1041.

Long, M., Rohde, H., \& Rubio-Fernandez, P. (2020). The pressure to communicate efficiently continues to shape language use later in life. Scientific Reports, 10, 1-13.

Lucas, C. G., Griffiths, T. L., Xu, F., Fawcett, C., Gopnik, A., Kushnir, T., Markson, L., \& Hu, J. (2014). The child as econometrician: A rational model of preference understanding in children. PloS one, 9, e92160. 
Mangold, R., \& Pobel, R. (1988). Informativeness and instrumentality in referential communication. Journal of Language and Social Psychology, 7, 181-191.

Nadig, A. S., \& Sedivy, J. C. (2002). Evidence of perspective-taking constraints in children's on-line reference resolution. Psychological Science, 13, 329-336.

Paraboni, I., \& Van Deemter, K. (2014). Reference and the facilitation of search in spatial domains. Language, Cognition and Neuroscience, 29, 1002-1017.

Paraboni, I., Van Deemter, K., \& Masthoff, J. (2007). Reference and the facilitation of search in spatial domains. Computational Linguistics, 33, 229-254.

Pogue, A., Kurumada, C., \& Tanenhaus, M. K. (2016). Talker-specific generalization of pragmatic inferences based on under-and over-informative prenominal adjective use. Frontiers in psychology, 6, 2035 .

Rehrig, G., Cullimore, R. A., Henderson, J. M., \& Ferreira, F. (2020). When more is more: Redundant modifiers can facilitate visual search. PsyArXiv, .

Repacholi, B., \& Gopnik, A. (1997). Early reasoning about desires: Evidence from 14-and 18-montholds. Developmental Psychology, 33, 12-20.

Rubio-Fernández, P. (2016). How redundant are redundant color adjectives? an efficiency-based analysis of color overspecification. Frontiers in psychology, 7, 153.

Rubio-Fernandez, P. (2019). Overinformative speakers are cooperative: Revisiting the gricean maxim of quantity. Cognitive science, 43, e12797.

Rubio-Fernandez, P. (2020). Redundant color words are more efficient than shorter descriptions. PsyArXiv, .

Rubio-Fernandez, P. (under review). The psychophysics of referential communication, .

Rubio-Fernandez, P., \& Jara-Ettinger, J. (2020). Incrementality and efficiency shape pragmatics across languages. $P N A S$, .

Rubio-Fernandez, P., Mollica, F., \& Jara-Ettinger, J. (2020). English and spanish speakers exploit language structure to increase communicative efficiency. Journal of Experimental Psychology: General, .

Ruby, P., \& Decety, J. (2001). Effect of subjective perspective taking during simulation of action: A pet investigation of agency. Nature Neuroscience, 4, 546-550.

Ryskin, R., Kurumada, C., \& Brown-Schmidt, S. (2019). Information integration in modulation of pragmatic inferences during online language comprehension. Cognitive Science, 43.

Sedivy, J. C. (2003). Pragmatic versus form-based accounts of referential contrast: Evidence for effects of informativity expectations. Journal of psycholinguistic research, .

Sedivy, J. C. (2005). 17 evaluating explanations for referential context effects: Evidence for cricean mechanisms in online language interpretation. Approaches to studying world-situated language use: Bridging the language-as-product and language-as-action traditions, (p. 345). 
Sonnenschein, S., \& Whitehurst, G. (1982). The effects of redundant communications on the behavior of listeners: Does a picture need a thousand words? Journal of Psycholinguistic Research, 11, 115-125.

Spivey, M. J., Tyler, M. J., Eberhard, K. M., \& Tanenhaus, M. K. (2001). Linguistically mediated visual search. Psychological Science, 12, 282-286.

Tourtouri, E., Delogu, F., Sikos, L., \& Crocker, M. (2019). Rational over-specification in visuallysituated comprehension and production. Journal of Cultural Cognitive Science, .

Viethen, J., van Vessem, T., Goudbeek, M., \& Krahmer, E. (2017). Color in reference production: the role of color similarity and color codability. Cognitive Science, 41, 1493-1514.

Westerbeek, H., Koolen, R., \& Maes, A. (2015). Stored object knowledge and the production of referring expressions: The case of color typicality. Frontiers in Psychology, 6.

Wimmer, H., \& Perner, J. (1983). Beliefs about beliefs: representation and constraining function of wrong beliefs in young children's understanding of deception. Cognition, 13, 103-128.

Zipf, G., \& Behavior, H. (1949). The principle of least effort. Reading, MA7 Addison Wesley, . 